Editorial

\title{
Pancreatic Cancer: Current Progress and Future Challenges
}

\author{
S. Perwez Hussain ${ }^{* \bowtie}$ \\ Head Pancreatic Cancer Unit, Laboratory of Human Carcinogenesis, Center for Cancer Research, National Cancer Institutes, NIH, Bethesda, MD 20892, USA \\ * Guest editor \\ $\triangle$ Corresponding author: hussainp@mail.nih.gov
}

(C) Ivyspring International Publisher. Reproduction is permitted for personal, noncommercial use, provided that the article is in whole, unmodified, and properly cited. See http://ivyspring.com/terms for terms and conditions.

Published: 2016.02.03

\begin{abstract}
Pancreatic ductal adenocarcinoma (PDAC), the most common form of pancreatic cancer, remains one of the highly lethal malignancies. The highly refractory nature of clinically advanced disease and lack of a reliable biomarker for early detection are major obstructions in improving patient outcome. The recent efforts, however, in understanding the pancreatic tumor biology have resulted in the recognition of novel addictions as well as vulnerabilities of tumor cells and are being assessed for their clinical potential. This special issue highlights some of the recent progress, complexity and challenges towards improving disease outcome in patients with this lethal malignancy.
\end{abstract}

Key words: Pancreatic Cancer

Pancreatic cancer is a highly lethal malignancy with a majority of the patients dying within a year after diagnosis and a median survival of less than 6 months (1). The lack of any specific and sensitive biomarker and asymptomatic nature of the disease make it harder to detect PDAC at an early stage, in addition to a complex biology and impenetrable tumor architecture, which presents a tough terrain to develop any effective treatment in advanced cases to improve disease outcome. In recent years, deciphering the complex tumor biology has exposed potential vulnerabilities of both tumor cells and its complex protective microenvironment, which can be exploited for therapeutic benefits. One example is reducing the stromal hyaluronic acid (HA) accumulation by using a HA-targeting enzymatic agent (PEGPH20), which reduced the interstitial fluid pressure and re-expanded the collapsed microvasculature and resulted in enhanced delivery of chemotherapeutic drug, gemcitabine, and survival in autochthonous mouse model of PDAC (2), and provided promising outcome in Phase 2 clinical trial. In the following col- lection of articles key issues have been discussed to understand, combat and ultimately defeat this lethal malignancy with focus on the current progress and future challenges to improve early detection and treatment strategies.

Therapeutic resistance is one of the major challenges in improving disease outcome in pancreatic cancer. Chand and colleagues discussed the potential mechanisms of therapeutic resistance in their article entitled "The landscape of pancreatic cancer therapeutic resistance mechanisms" (7) and highlighted the potential targets to overcome the highly stubborn resistance and enhance drug efficacy in pancreatic cancer. They described hypoxia, among others, as one of the major events that contributes to the therapeutic resistance and can be mediated by HIF-dependent and -independent mechanisms. One of the HIF-1a independent mechanisms is the stabilization of PIM1 (proviral integration site for moloney murine leukemia virus 1), a serine/threonine kinase, by $\mathrm{HuR}(\mathrm{Hu}$ antigen R), which is translocated to the cytoplasm following hypoxia. Additionally, post-transcriptional 
regulation, primarily by RNA binding proteins, e.g., HuR, and microRNA plays a role in therapeutic resistance, and several other studies utilizing synthetic lethality with PARP inhibition are discussed that underscores their potential as therapeutic targets for overcoming resistance and enhancing drug efficacy.

Smith et. al., focused on the role of gastrin and cholecystokinin (CCK) receptors in the development and progression of pancreatic cancer (8). Although gastrin is expressed in embryonic pancreas and not in the adult, an increased expression of gastrin is found in pancreatic cancer. Activation of CCK receptors by its ligands, gastrin or CCK, may induce several oncogenic signaling pathways including PI3K/AKT, MAPK and mTOR. Animal studies have provided proof-of-concept that targeting gastrin, CCK receptors, and their interaction may have potential therapeutic significance. Future clinical studies exploiting the gastrin-CCK receptor interface would further determine the significance of this strategy in improving patient outcome.

Diagnosis of resectable early-stage pancreatic cancer, and its surgical resection followed by adjuvant therapy confer 5 -year survival only in about $20 \%$ of the resected patients and a large percentage of patients show recurrence within 2 years after surgery. It is argued that identification of biomarkers associated with established precursor lesions of pancreatic cancer is critical for early detection and treatment (3). Consistent with this notion, Guo et. al., have described the molecular biomarkers of pancreatic intraepithelial neoplasia (PanINs), a well-established precursor lesion in pancreatic ductal adenocarcinoma (PDAC), and discussed their potential significance in early diagnosis and treatment (9).

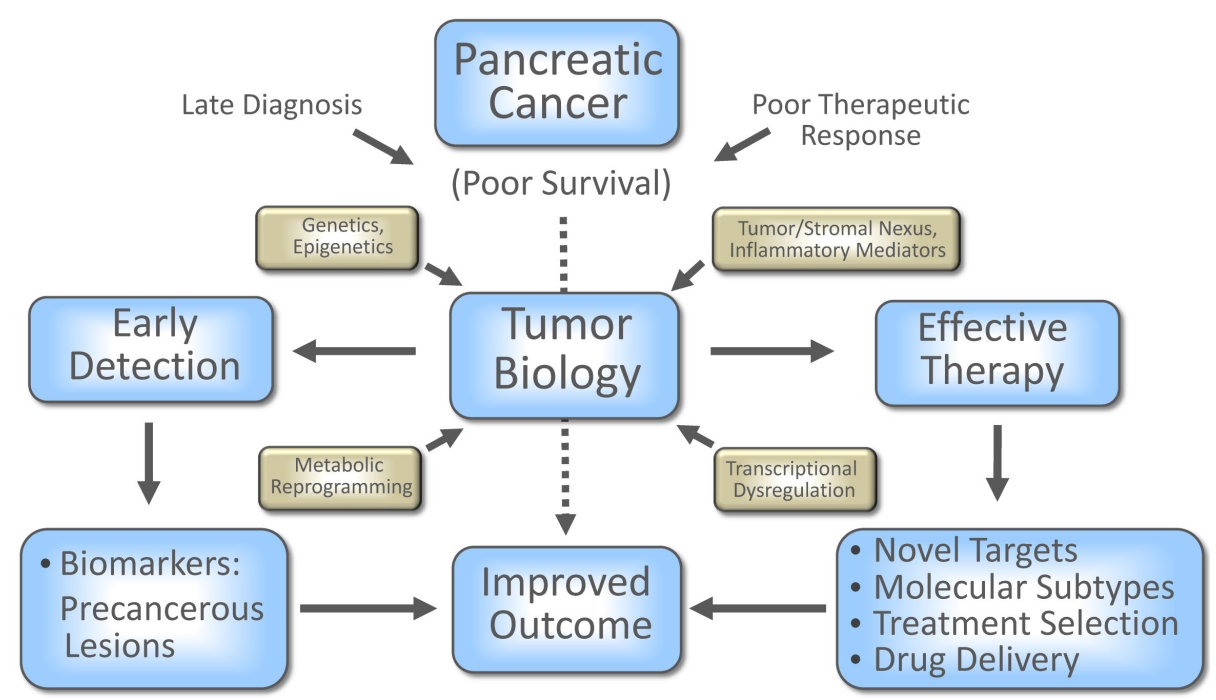

Figure 1: Recent advances in understanding pancreatic tumor biology and the underlying mechanism of disease aggressiveness are providing clues, which may help to potentially improve diagnosis, treatment and patient outcome. Some of the critical biological determinants in the development and progression of pancreatic cancer have been reviewed in this special issue.
A massive, highly reactive, desmoplastic stroma, consisting of fibroblasts, immune and inflammatory cells, endothelial cells and extracellular matrix characterize PDAC. Evidence of a strong tumor-stromal interaction in the progression of pancreatic cancer has led to the recent efforts of targeting stromal components to inhibit pancreatic tumor progression. In a recent study, $\mathrm{T}$ cells engineered to express affinity enhanced $\quad T$ cell receptors against mesothelin evaded stromal barriers, lysed tumor cells, and enhanced survival in mice with PDAC (4). Aligned with these immune modulating strategies, Felix and Gaida discuss the role of polymorphonuclear neutrophils (PMN) and PMN-derived proteases, in particular, in the progression of PDAC and highlight the therapeutic opportunities of targeting the interactive pathways between PMN-derived proteases, stromal components and tumor cells (10).

A number of epidemiological risk factors have been linked to pancreatic cancer, including smoking, type 2 diabetes, obesity and chronic pancreatitis (5). About $5-10 \%$ of pancreatic cancer is linked to the family history and hereditary cancer syndromes, for example, Peutz-Jeghers syndrome, hereditary pancreatitis, hereditary nonpolyposis colorectal cancer, and familial atypical multiple mole and melanoma syndrome, that are characterized by mutation/alteration in specific genes. Additionally, genome wide association studies (GWAS) have identified a number of common low risk pancreatic cancer susceptibility loci. Along these lines, Laufey Amundadottir in her article entitled "Pancreatic Cancer Genetics" has provided an in-depth description of the germline risk variants for pancreatic cancer with special focus on common susceptibility alleles identified by GWAS. Several ongoing studies on the mechanistic and functional roles of these germline variants and susceptibility loci would help in designing diagnostic, preventive and therapeutic strategies for pancreatic cancer (11).

Altered expressions of microRNAs (miRNA) are found in PDAC. Several of these microRNAs have been associated with prognosis in PDAC patients and their mechanistic and functional roles in the development and progression of pancreatic cancer have been examined in human pancreatic cancer cells and preclinical in vivo models. 
Among these, several miRNAs, for example mir-21, mir-221, miR-155, are found to have oncogenic functions, while mir-34a/b, Let-7, mir-146a displayed tumor suppressive activities in pancreatic cancer. $\mathrm{Li}$ and Sarkar in their article entitled "microRNA targeted therapeutic approach for pancreatic cancer" reviewed the role of miRNAs in pancreatic cancer and described the current understanding on their potential as biomarkers for early detection, prognosis and monitoring the treatment outcome, and as therapeutic targets (12).

Logsdon and Lu in their article "The significance of Ras activity in pancreatic cancer initiation" discuss the importance of a high and sustained activity of oncogenic Ras to attain a pathological level, which is achieved by inflammatory mediators in the initiation of pancreatic cancer, different from the current paradigm, which suggests that oncogenic KRas is constitutively active to a level sufficient for tumorigenesis (13). In a recently published study, Logsdon and colleagues have shown that KRas activity, upon exceeding a threshold level, induces the generation of inflammatory mediators that maintains the pathological level of Kras in a positive feedback manner. By presenting a KRas/inflammation feed-forward model of pancreatic cancer initiation, an alternative paradigm has been, therefore, put forward for the role of oncogenic KRas in tumor initiation in which strategies targeting inflammatory mediators/pathways may be pursued to diminish KRAS oncogenic function (6).

These articles have addressed both the biological complexity as well as vulnerability of pancreatic cancer. The advancement in our understanding of the tumor biology and underlying mechanism of disease aggressiveness have offered many attractive candidate biomarkers for early detection, and potential therapeutic targets for designing novel treatment strategies. Hopefully, not in too distant future, some of this knowledge will be translated into clinical use for improving survival in patients with this lethal malignancy.

\section{Acknowledgements}

This work is supported by the intramural research program of the Center for Cancer Research, NCI, NIH.

\section{References}

1. Siegel RL, Miller KD, Jemal A. Cancer statistics, 2015. CA Cancer J Clin. 2015;65:5-29.

2. Provenzano PP, Cuevas C, Chang AE, et al. Enzymatic targeting of the stroma ablates physical barriers to treatment of pancreatic ductal adenocarcinoma. Cancer Cell. 2012;21:418-29.

3. Goggins M. Markers of pancreatic cancer: working toward early detection. Clin Cancer Res. 2011;17:635-7.

4. Stromnes IM, Schmitt TM, Hulbert A, et al. T Cells Engineered against a Native Antigen Can Surmount Immunologic and Physical Barriers to Treat Pancreatic Ductal Adenocarcinoma. Cancer Cell. 2015;28:638-52.
5. Vincent A, Herman J, Schulick R, et al. Pancreatic cancer. Lancet. 2011;378:607-20.

6. Daniluk J, Liu Y, Deng D, et al. An NF-kappaB pathway-mediated positive feedback loop amplifies Ras activity to pathological levels in mice. J Clin Invest. 2012;122:1519-28.

7. Chand S, O'Hayer K, Blanco FF, Winter JM, Brody JR. The Landscape of Pancreatic Cancer Therapeutic Resistance Mechanisms. Int J Biol Sci 2016; 12(3):273-282. doi:10.7150/ijbs.14951.

8. Smith JP, Fonkoua LK, Moody TW. The Role of Gastrin and CCK Receptors in Pancreatic Cancer and other Malignancies. Int J Biol Sci 2016; 12(3):283-291. doi:10.7150/ijbs.14952.

9. Guo J, Xie K, Zheng S. Molecular Biomarkers of Pancreatic Intraepithelial Neoplasia and Their Implications in Early Diagnosis and Therapeutic Intervention of Pancreatic Cancer. Int J Biol Sci 2016; 12(3):292-301. doi:10.7150/ijbs.14995.

10. Felix K, Gaida ${ }^{2}$ MM. Neutrophil-Derived Proteases in the Microenvironment of Pancreatic Cancer -Active Players in Tumor Progression. Int J Biol Sci 2016; 12(3):302-313. doi:10.7150/ijbs.14996.

11. Amundadottir LT. Pancreatic Cancer Genetics. Int J Biol Sci 2016; 12(3):314-325. doi:10.7150/ijbs.15001.

12. Li Y, Sarkar FH. MicroRNA Targeted Therapeutic Approach for Pancreatic Cancer. Int J Biol Sci 2016; 12(3):326-337.

13. Logsdon CD, Lu W. The Significance of Ras Activity in Pancreatic Cancer Initiation. Int J Biol Sci 2016; 12(3):338-346. doi:10.7150/ijbs.15020. 